\title{
Community reintegration and quality of life following spinal cord injury
}

\author{
M J DeVivo DrPH, J S Richards PhD \\ Department of Rehabilitation Medicine, University of Alabama at Birmingham, \\ Birmingham, Alabama 35233-7330, USA.
}

\begin{abstract}
This report contains a brief overview of issues related to community reintegration and quality of life for persons with spinal cord injuries. Current data from the National Spinal Cord Injury Statistical Center are provided on place of residence, employment, education and marital status after rehabilitation discharge. The health policy implications of these data are discussed along with prospects for continued improvement of long term social outcomes and directions for future research.
\end{abstract}

Key words: spinal cord injury; employment; education; marital status; residence.

\section{Introduction}

The long term goal of spinal cord injury (SCI) rehabilitation is to achieve community reintegration with the maximum possible level of functional independence and a return to preinjury lifestyle. This paper provides the latest information from the US model regional SCI care systems that is contained in the National Spinal Cord Injury Statistical Center (NSCISC) database. The history of the model system program and its data collection protocol have been described elsewhere. ${ }^{1,2}$

The NSCISC database includes 3 objective measures of community reintegration and lifestyle: place of residence, employment status, and marital status. We will consider each of these and conclude with a general discussion of quality of life issues and future prospects for SCI research in these areas.

\section{Place of residence}

Persons who complete rehabilitation are almost always (94.1\%) discharged to a private residence within the community. Since 1973 , only $4 \%$ of all persons treated at model systems of care were discharged to a nursing home. ${ }^{1}$ Moreover, $98 \%$ of all living persons with SCI initially treated at model systems still live in private residences 10 years after injury. ${ }^{1}$ Not surprisingly, age is strongly correlated with place of residence. Among persons between 16 and 30 years of age, only $1.4 \%$ reside in nursing homes, whereas the corresponding figure for persons over age 75 is $22.2 \% .^{3}$ Therefore, since improving life expectancies are resulting in increasing numbers of elderly persons with SCI, additional attention should be focused on alternative means of providing needed services for these individuals.

The enormous success in returning persons with SCI to private residences following rehabilitation is, in part, due to the efforts of the growing and highly vocal independent living movement. In the future, however, given the extremely high costs of skilled nursing and attendant care necessary for more severely injured persons to live in private residences, third party payers may attempt to place more persons with SCI into nursing homes where costs could be substantially reduced. Therefore, to ensure continuing success in returning persons with SCI to private residences, we must continue to document the considerable benefits of independent living.

\section{Employment}

Among persons with SCI between the ages of 16 and 59, the percentage employed in 
the competitive labor market increases from $12.6 \% 2$ years after injury to $38.3 \% 12$ years after injury. ${ }^{1}$ Significant predictors of postinjury employment include younger age, being male, being white, having a higher educational level, being highly motivated, having sufficient functional ability, and being employed prior to the injury. ${ }^{4}$

Persons employed shortly after injury virtually always return to the same job and employer they had prior to injury. Conversely, persons whose labor market entry is delayed at least a few years usually obtain a different job with a different employer. ${ }^{5}$ For example, at the time of their injury, few persons $(28.3 \%)$ are employed in professional, clerical and sales jobs. Four years after injury, however, $63 \%$ of employed persons with paraplegia and $78 \%$ of employed persons with quadriplegia have these jobs. ${ }^{5}$ Continuing availability of vocational rehabilitation services, therefore, will be important to facilitate retraining or further education required for persons with SCI to return to work.

Many persons whose labor market entry is delayed have dropped out of school at an early age or are students who first need to complete their formal education at high school and college levels. Among persons injured between the ages of 18 and 21, 67\% have at least a high school diploma, somewhat less than the nationwide norm for persons without SCI of $78 \% .{ }^{1}$ Model system data reveal that $51.2 \%$ of persons with a 9 th to 11th grade education at the time of their injury successfully finish high school, and $11.6 \%$ of persons with a high school diploma achieve a higher academic degree within the first 5 years after injury. ${ }^{1}$ Undoubtedly, there are more persons who complete their educations beyond this time period. Increasing these percentages is a critical step toward increasing employment options after SCI.

A disturbing trend has occurred in recent years. As a result of funding cutbacks, the percentage of persons who become clients of state Departments of Vocational Rehabilitation has declined from $52.4 \%$ in 1974 to only $26.4 \%$ in 1990 . Given the low education levels and need for job retraining, we must attempt to return vocational reha- bilitation activities to previous funding levels before employment rates also decline.

Strong economic disincentives to employment also exist following SCI. ${ }^{6-7}$ These disincentives must be eliminated and replaced by financial inducements to return to work.

Other reasons for the low employment rate following SCI include discriminatory hiring practices, architectural and attitudinal barriers, and transportation difficulties. The recently enacted Americans with Disabilities Act (Public Law 101-336) was a step in the right direction, but will not solve these problems overnight. Progress in these areas will likely continue at a slow pace.

Finally, it is important to recognize that competitive labor market employment is not an appropriate goal for every working age person with SCI. Some individuals are too severely injured to return to competitive employment while others are financially independent as a result of litigation and, therefore, quite understandably lack motivation to return to work. For those who are homemakers or engaged in volunteer activities at the time of their injury, the appropriate goal is often a return to those activities. Therefore, successful community reintegration should be assessed using a broad definition of 'gainful employment' that includes all productive activities.

\section{Marital Status}

SCI has at least a short term impact on marriage and divorce rates. ${ }^{1,8-10}$ Among never married persons treated at model systems, $12 \%$ get married within the next 5 years, whereas $34.7 \%$ would be expected to get married based on rates for the general population of comparable age, gender and race. ${ }^{1}$ Further research is needed to determine whether expected marriages are merely being postponed beyond this time frame or persons with SCI are remaining single indefinitely.

The impact on divorce rates is less dramatic. Among married persons at the time of their injury, $80.7 \%$ were still married 5 years later, compared to an expected $88.8 \%{ }^{1}$ Among postinjury marriages, $56 \%$ 
are still intact 8 years later, whereas $77 \%$ were expected to remain intact based on divorce rates for a demographically comparable general population.

Significant risk factors for divorce include young age, being female, being black, having no children, having previous marriages that ended in divorce, and being nonambulatory. ${ }^{8}$ Interestingly, although the long term picture is less clear, it appears that after a period of high risk lasting a few years, the divorce rate for persons with SCI begins to decline toward the normal rate for the general population. ${ }^{1}$ Moreover, marriages that do survive are generally viewed as satisfying and comfortable. ${ }^{11}$

\section{Quality of life}

With continuing improvements in survival, SCI researchers are becoming more interested in quality of life, a somewhat elusive concept which of necessity is defined by the subjective experience and report of the person who lives with this disability. This line of investigation is being partially driven by the curiosity and concern of health professionals about whether persons with SCI are able to return to what they define as a quality life and are, therefore, grateful for their survival. As reflected by the relatively high suicide rate, some clearly are not. ${ }^{12}$ However, the SCI population is not demographically and psychosocially the same as the ablebodied population; a high percentage of preinjury and postdischarge substance abuse, for example, has been documented. ${ }^{13}$ Therefore, it could turn out that the SCI suicide rate is actually not above normal. The increased interest in medical ethics, the right to die, living wills, etc, have been spurred by the considerable media attention focusing on individuals who survive SCI and then choose to terminate their lives. ${ }^{14,15}$ Individual cases can be instructive but cannot be used to generalize. What group data are there, then, that shed light on the issue of quality of life which, in its absence, encourages some individuals to terminate their lives?

The bulk of evidence to date suggests that most people after SCI are able to achieve what they define as a satisfactory quality of life. During the first year postdischarge, there is evidence of decreasing depression and hostility over time. ${ }^{16}$ Beyond the first year, there is evidence of increasing acceptance of disability and improving life satisfaction as time postinjury increases. ${ }^{17-20}$ Interestingly, level or extent of SCI do not predict life satisfaction; persons with more physically limiting injuries do not necessarily report less satisfaction with life. ${ }^{21}$

The question then becomes: if life satisfaction is not correlated with extent of disability, what factors explain it? And, if predictors of life satisfaction can be found, can points of intervention also be identified? Recent researchers have utilized the stress/ coping theoretical rationale for investigating postdischarge adjustment. ${ }^{17,22-24}$ Such approaches involve examining different methods of coping with stress as well as mediating and moderating factors that facilitate coping. Although the important role of social support in facilitating SCI adjustment has already been demonstrated, ${ }^{1 \dagger}$ the key components of social support have not been defined, and factors that affect the ability of caregivers to provide effective support have not been identified.

The Americans with Disabilities Act, referred to earlier, coupled with prior legislation (eg Public Law 94-142), should provide increased leverage to assist persons with SCI and other disabling conditions gain full access to life opportunities by minimizing barriers to work, education, housing and transportation. The extent that these legal changes impact quality of life for persons with SCI remains to be seen. Quality of life is a product of the interaction of personal attributes and resources with environmental resources. Legislated improvements in the physical receptiveness of the environment to persons with disabilities are best seen as necessary, but not sufficient for achieving an adequate quality of life. Attitudes of others and personality correlates of effective coping cannot be legislated. However, attitudes can be changed and coping styles altered. Over the next decade, we can expect increased efforts to develop interventions on personal, social and environmental levels designed to yield improvements in quality of life for persons with SCI. 


\section{Conclusions}

Advances in medical management have allowed improved survival following SCI. Much work remains to be completed, however, to carry these advances further. Even more research focusing on long term complications and adjustment is needed. Limited available data suggest a relatively optimistic picture. Persons discharged from the model SCI systems of care are virtually always discharged to a private home. Marital status and divorce rates appear to be impacted by SCI, although not as much as some have predicted. An increasing percentage of persons with SCI are able to return to competitive employment or homemaking over time, and this is often facilitated by vocational rehabilitation support and further formal education. These data do not reflect very directly, however, impact of SCI on perceived quality of life. Certainly the efforts of patient, family and rehabilitation personnel would seem somehow unfruitful if a reasonable quality of life is not achieved. Data are now being gathered that reflect perceived satisfaction with quality of life. Individual cases not withstanding, it appears that community reintegration is also accompanied by a perceived high quality of life for most persons with SCI and their significant others. Future prospective studies are needed which delineate better the correlates of effective coping and point toward interventions targeted at those who do not move ahead effectively with life.

\section{Acknowledgements}

Preparation of this manuscript was supported in part by grant No. H133N00001 from the National Institute on Disability and Rehabilitation Research (NIDRR), Department of Education, Washington DC, USA.

\section{References}

1 DeVivo MJ, Richards JS, Stover SL, Go BK (1991) Spinal cord injury-rehabilitation adds life to years. West J Med 154: 602-606.

2 Stover SL, Fine PR (1986) Spinal Cord Injury: The Facts and Figures. University of Alabama at Birmingham.

3 DeVivo MJ, Black KJ, Go BK, Stover SL (1991) Relationship between age and current health status for persons with spinal cord injury. Arch Phys Med Rehabil 72: 774.

4 DeVivo MJ, Rutt RD, Stover SL, Fine PR (1987) Employment after spinal cord injury. Arch Phys Med Rehabil 68: 494-498.

5 Young JS, Burns PE, Bowen AM, McCutchen R (1982) Spinal Cord Injury Statistics: Experience of the Regional Spinal Cord Injury Systems. Good Samaritan Medical Center, Phoenix, Arizona.

6 Better SR, Fine PR, Simison D, Doss GH, Walls RT, McLaughlin DE (1979) Disability benefits as disincentives to rehabilitation. Milbank Mem Fund $Q$ 57: 412-427.

7 Walls RT, Masson C, Werner TJ (1977) Negative incentives to vocational rehabilitation. Rehabil Lit 38: $143-150$.

8 DeVivo MJ, Fine PR (1985) Spinal cord injury: its short-term impact on marital status. Arch Phys Med Rehabil 66: 501-504.

9 DeVivo MJ, Jenkins KD, Go BK (1989) Outcomes of post-spinal cord injury marriages. Arch Phys Med Rehabil 70A: 50.

10 Brown JS, Giesy B (1986) Marital status of persons with spinal cord injury. Soc Sci Med 23: 313-322.

11 Crewe NM, Krause JS (1988) Marital relationships and spinal cord injury. Arch Phys Med Rehabil 69: 435-438.

12 DeVivo MJ, Black KJ, Richards JS, Stover SL (in press) Suicide following spinal cord injury. Paraplegia.

13 Heinemann A, Donohue R, Keen M, Schnoll S (1988) Alcohol use by persons with recent spinal cord injuries. Arch Phys Med Rehabil 69: 619-624.

14 Maynard FM, Muth AS (1987) The choice to end life as a ventilator-dependent quadriplegic. Arch Phys Med Rehabil 68: $862-864$.

15 Mourer, S.A. (1991) The refusal of treatment: staff reaction and recommendations. SCI Psychosocial Process 3(4): 72-76.

16 Richards JS (1986) Psychologic adjustment to spinal cord injury during the first postdischarge year. Arch Phys Med Rehabil 67: 362-365.

17 Schulz R, Decker S (1985) Long-term adjustment to physical disability: the role of social support, perceived control, and self-blame. J Pers Soc Psychol 48: 1162-1172.

18 Woodrich F, Patterson JB (1983) Variables related to acceptance of disability in persons with spinal cord injuries. J Rehabil 49: 26-30.

19 Krause JS, Crewe NM (1991) Chronologic age, time since injury, and time of measurement: effect on 
adjustment after spinal cord injury. Arch Phys Med Rehabil 72: 91-100.

20 Lundquist C, Siosteen A, Blomstrand C, Lind B, Sullivan M (1991) Spinal cord injuries: clinical, functional and emotional status. Spine 16: 78-83.

21 Siosteen A, Lundquist C, Blomstrand C, Sullivan L, Sullivan M (1990) The quality of life of three functional spinal cord injury subgroups in a Swedish community. Paraplegia 28: 476-488.

22 Elliott TR (1991) Interpersonal behavior and adjustment of persons with spinal cord injury. SCI Psychosocial Process 3: 82-87.

23 McColl MA, Friedland J Development of a multidimensional index for assessing social support in rehabilitation. Occup Ther Res 9: 218-234.

24 Nieves CC, Charter RA, Aspinall MJ (1991) Relationship between effective coping and perceived quality of life in spinal cord injured patients. Rehabil Nurs 16: 129-132. 